\title{
Iatromechanik und Chirurgie bei Lorenz Heister
}

Von Urs Boschung

\section{Heister als Iatromechaniker}

Es mag vielleicht erstaunen, Lorenz Heister (1683-1758), den bekanntesten deutschen akademischen Chirurgen der ersten Hälfte des 18. Jahrhunderts, als Theoretiker der Medizin behandelt zu sehen ${ }^{1}$. In der Tat war die Geschichtsschreibung der Chirurgie an diesem Aspekt bisher nicht sehr interessiert. Ich möchte jedoch zeigen, daß die mechanistische Grundposition dem Arzt des ausgehenden 17. und beginnenden 18. Jahrhunderts die Beschäftigung mit der Chirurgie erleichterte. Lorenz Heister bietet dazu gute Gelegenheit, da er für einen Doktoranden, Johann Peter Sparward aus Stralsund, 1738 in Helmstedt eine Dissertatio de medicinae mechanicae praestantia, (deutsch: Von der Fürtrefflichkeit und dem Fürzuge der mechanischen Artzney-Lehre) verfaßte ${ }^{2}$. Diese Dissertation erschien später unter Heisters alleinigem Namen als Einleitung zum Compendium medicinae practicae, Amsterdam 1743 (deutsch: Practisches Medicinisches Handbuch, Leipzig 1744); sie ist in erster Linie als Kampfschrift gegen Georg Ernst Stahl (1660-1734) und den von ihm und seinen Anhängern vertretenen sog. Animismus zu verstehen ${ }^{3}$.

Heister beginnt seine Abhandlung mit einem Überblick über die Geschichte der medizinischen «Secten». Gegenwärtig seien besonders in Deutschland noch zwei im Schwange, nämlich die «Mechanische (worunter ich zugleich die Hippocratische oder vernünftige verstehe)» und die Stahlianische. Heister definiert die «mechanische Medicin» als

«diejenige Artzney-Wissenschaft, da man aus der des weisen Schöpfers so künstlichen Zubereitung und Zusammensetzung des menschlichen Leibes, wie wir in der ZergliederungsKunst lernen, und denen daraus fließenden Würckungen der festen und flüßigen Theile, durch physicalische, mechanische und chemische Anmerckungen und Erfahrungen, nach den mechanischen Grund-Sätzen untersuchet und erkläret. Denn der menschliche Cörper ist nichts anders als eine sehr künstliche Maschine, oder Gebäude, welche wiederum aus vielen kleinen Maschingen bestehet. Dieses beweiset sowohl die Zergliederungs-Kunst, als auch der berühmte Boerhave in seinen Institutionibus Medicis und noch viel andere medicinische mechanische Scribenten ${ }^{4}$..., welche sich das Joch ... [der Autorität] vom Halse geschüttelt, und nichts für wahr erkannt haben, als was sie mit den Sinnen durch gewisse Erfahrungen und Anmerckungen haben begreifen und beweisen können, das ist, aus der Beschaffenheit unsers 
Cörpers sowohl im gesunden als krancken Zustande durch gewisse Vernunft-Schlüsse haben herleiten und darthun können» (§2).

\section{Die Gegenposition Stahls und seiner Anhänger formuliert Heister wie folgt:}

«Sie halten nehmlich die Seele und die Natur des Menschen für eins, und wollen daraus alles, was sowohl zur Erlernung als Ausübung der Artzeney gehöret, erklären. Denn die vernünftige Seele ist, nach ihrer Meynung, eben dasjenige, welches den Leib der Frucht im Mutter-Leibe baue; die Seele sey es ferner, welche in dem zubereiteten und gebohrnen Menschen auf alle Würckungen desselben achtung gebe, und Sorge trüge, daß dieser Cörper, als ihr Wohn-Hauß, gesund und unverletzt erhalten würde, daher sie auch alle so genannte Seelische, Lebens- und natürliche Würckungen im Cörper regiere; ja wenn sie einen Fehler in dem Cörper zu seinem Nachtheil gewahr würde, so bemühe sie sich auf alle Art und Weise dieses Schädliche zu verbessern oder aus dem Leibe herauszuwerfen; und deswegen berathschlage die Seele bey Zeiten, bey welchen das Brechen, oder bey andern der Stuhlgang, der Schweiß oder der Urin zu erregen dienlich sey; bey andern wiederum verursache sie Blut-Flüsse, durch die Nase, Lunge, durch den Magen, Nieren, Blase, oder durch den Mast-Darm, welche man die güldene Ader nennet, also schaffe sie das überflüßige oder schädliche zur Gesundheit des Leibes aus dem Wege; bey andern ziehe sie die Theile zusammen durch Krampf und Schmertzen, und suche auf diese Weise das schädliche aus gewissen Theilen heraus oder an andere Orte zu treiben ..., und alsdenn verändere sie die schädliche Materie entweder in Schleim ... oder in Eyter ...» (§ 4).

\section{Heister bemerkt weiter,}

«daß die Stahlianer nicht ... [so sehr] die Unreinigkeit des Geblüts, nehmlich der Chemicorum ihre saltzige Schärfe, nehmlich das Saure und Alcali, als die Ursache vieler Kranckheiten ansehen, sondern vielmehr den $\ddot{U}$ berflu $\beta$ des Geblüts, welchen man insgemein die Vollblütigkeit oder Plethoram nennet, nicht unrecht anklagen. Und daher sagen sie, daß sich die Seele in den meisten Kranckheiten eyfrigst dahin bemühe, daß sie den Cörper durch erregte freywillige BlutFlüsse oder Schleim-Auswürfe von dem Überflusse befreye, davon die meisten Beschwerungen herrührten ...» $(\S 6)$.

\section{Unter Berufung auf Hippokrates raten Stahl und seine Anhänger,}

«daß ein Medicus bey allen Kranckheiten wohl achtung geben solle, wo die Natur oder die Seele hin wolle, da müsse er ihr folgen; denn die Natur sey der beste Artzt, der Medicus aber nur ein Diener derselben; und derjenige sey der beste Medicus, welcher gedachte Bewegungen wohl beobachte, und der Natur in ihren Absichten folge und beförderlich sey» (§5).

Heister beginnt seine Widerlegung der Stahlschen Lehre mit einer Kritik des Seelenbegriffs. Unter Berufung auf Descartes sieht er in der Seele ein «denckendes Wesen» und folgert daraus, die Seelentätigkeiten müßten dem Bewußtsein offenbar sein. Dies treffe für die willkürlichen Bewegungen und für die Sinnestätigkeiten zu, nicht aber für die Verrichtungen des Herzens 
oder der Verdauung, ebenso wenig für die Absonderung der Säfte usw. Wer trotz des fehlenden Bewußtseins diese Funktionen der Seele zuschreibe, falle in das Labyrinth der «qualitates occultae» zurück, die doch Descartes und die «Mechanici» aus der Medizin zu vertreiben sich bemüht hätten (§ 7). In der Naturforschung dürfe nichts als wahr oder sicher angenommen werden, was nicht durch Experimente und Beobachtung (vel experimentis vel sensibus) bestätigt sei (§8). Im Gegensatz zu Stahls Behauptung lehrt vielfältige Erfahrung vielmehr:

«... es verrichten alle diese Theile ihre Würckungen nicht anders als sehr künstliche Wercke oder Maschinen, oder als ein Uhr-Werck, als eine Mühle und dergleichen, welche bloß allein durch den Willen des Menschen ohne äußerlich angewandte Gewalt sich nicht verändern, und weder geschwinder noch langsamer gehen. Denn sie können nicht anders würcken, als es der Bau, das Gemächte, die Gestalt und die Zusammensetzung ihrer Theile [fabrica sive mechanica constructio] mit sich bringet, und dis so lange, bis sie von einer andern äußerlichen Gewalt oder Ursache daran gehindert werden» (§ 10).

Entsprechend dem radikalen Dualismus von Descartes haben Leib und Seele - abgesehen von den mit Willen und Bewußtsein verknüpften Tätigkeiten - nichts miteinander zu schaffen. Wie die Seele in den genannten Fällen mit dem Körper in Beziehung tritt, ist unerklärbar ${ }^{5}$.

Heister legt nun ausführlich die Grundzüge einer mechanistischen Physiologie dar, die sich auf die Solida und die Fluida, die zwei Grundbestandteile des Körpers, stützt und die mit zahlreichen Modellen aus Mechanik und Technik deren Funktionen erklärt (§ 13-29).

Im Abschnitt Pathologie (§30-50) findet es Heister mit den Eigenschaften eines denkenden, sinnvoll handelnden Wesens unvereinbar, daß die Seele zur Überwindung der Plethora so gefährliche Ereignisse wie den Blutsturz verursachen soll. Ähnliches gilt für die Fieber, die nach der Meinung der Mechaniker durch Verstopfung der kleinen Gefäße durch Galle, Schleim u.a.m. entstehen.

Besonderer Erörterung bedarf der in der Medizin seit Alters her beliebte Begriff der «Heilkraft der Natur». ${ }^{6}$ Die Stahlianer setzen Natur mit Seele gleich; Heister vermutet, daß Unkenntnis der Anatomie daran schuld ist; sonst wüßten sie,

«daß unser Cörper also von Gott gebauet sey, daß er sich selbst oft nicht nur sehr lange gesund erhält, sondern sich auch in vorfallenden Kranckheiten durch seine eigene ihm von Gott verliehene Kräfte, das ist, durch pur natürliche und mechanische Ursachen, ohne Hülfe der Seele, wieder helfen kan, gleichwie bey Thieren» (§49). 


\section{Die Natur des Menschen ist demnach}

«nichts anders als der künstlich mechanisch-eingerichtete Bau oder Gemächte [fabricam illam artificiosissimam mechanicam] des Cörpers ..., in welchem die flüßigen Theile von den vesten, und diese wieder von jenen auf eine wunderbare Weise in eine beständige abwechselnde Bewegung gesetzet werden» (§50).

In der Praxis ist es falsch, ohne Rücksicht auf die Umstände immer der «Diener der Natur» sein zu wollen, wie es die Stahlianer fordern. Manchmal ist es notwendig, der Natur entgegenzuwirken ${ }^{7}$, bei Blutstürzen, Pneumonie usw. Heister vergleicht den Arzt mit dem Ingenieur:

«Denn gleichwie gute Ingenieurs [boni Mathematici] starcke Wasser-Güsse und Überschwemmungen auf allerhand Weise abzuleiten suchen, damit es denen Gebäuden, Mauren, Wällen, ja gantzen Schlössern einer Stadt oder eines Landes keinen Schaden ... zufügen könne; also machen es auch vernünftige mechanische Medici, welche ... böse Feuchtigkeiten vom Schlunde, von den Ohren oder Augen, welchen sie Gefahr, Schmertzen, Abscesse und dergleichen drohen, auf andere Wege zu leiten und auszuführen trachten» (§ 58$)$.

Für den Bereich der praktischen Medizin hat sich Heister die Chirurgie als stärkstes Argument aufgespart:

«Es zeiget sich aber der Fürzug der mechanischen Artzney-Kunst nirgends mehr als in demjenigen allerältesten und fürtreflichen Theile derselben, welchen man die Chirurgie nennet, indem alles, was man in derselben vornimmt, auf mechanische Art geschiehet, und auch nicht anders geschehen kan, dabey man die Ohnmacht der Stahlianischen Seele am meisten erkennet» (§65).

Frakturen, Luxationen, Wunden, Blutungen, Atresien und Spaltmißbildungen, Fisteln, Ergüsse usw. werden einzig und allein durch mechanische Maßnahmen geheilt. (Auffälligerweise läßt Heister hier die Geburtshilfe unerwähnt!)

\section{Heister faßt zusammen $(\S 66)$ :}

1. Positive, gesicherte Erkenntnis ist in der Medizin nur durch Erforschung des Baues des Körpers und des Zusammenhangs, der Einrichtung, Lage und Wirkung seiner Teile möglich. Alles, was hier bekannt ist, verdankt man den «mechanischen Medicis».

2. Daraus ergibt sich, «daß alle Kranckheiten im menschlichen Cörper aus mechanischen Ursachen entspringen». "Sympathetische» und andere Kuren, «welche nicht auf mechanische Gründe gebauet sind», erweisen sich als «meistens gantz falsch, oder wenigstens sehr ungewiß». 
3. Die mechanistische Naturlehre hat «zur Verherrlichung des Schöpfers» dargetan, «daß die künstliche Maschine unsers Cörpers von ihm so wunderbar und weislich gemachet sey, daß sich selbige nicht nur selbst ohne Hülfe der Seele sehr lange erhalten, sondern sich auch durch ihre eigene Natur in Kranckheiten wieder helfen ... könne ...»

4. Die mechanischen Ärzte haben viele und vortreffliche chirurgische Instrumente und Heilmethoden erfunden, wohne welche die practische Artzney-Kunst sehr mangelhaft, und viele Patienten elend und ungesund bleiben würden. Es bezeugen solches sowohl die alten als neuern Schriftsteller, welche von der Chirurgie geschrieben und ihre Werkzeuge haben abzeichnen lassen, welches in der That mechanische Medici gewesen.»

\section{Iatromechanik und Chirurgie}

Lorenz Heister ist in erster Linie bekannt als Verfasser der Chirurgie und des Compendium anatomicum; seine weiteren Schriften werden weniger gewürdigt. Heister beanspruchte jedoch für sich als gelehrten Arzt und Universitätsprofessor, das Gesamtgebiet der Medizin (einschließlich der Botanik) zu überblicken und zu lehren, wie sein umfangreiches Schriftenverzeichnis genügend belegt ${ }^{8}$.

Auch als Chirurg geht Heister von theoretischen Grundlagen aus. Die mechanistische Konzeption der Lebensvorgänge ist geradezu Voraussetzung und Legitimation für die Beschäftigung mit der Chirurgie. Als ehemaliger Leidener Student steht Heister in der Tradition der berühmten Rede Boerhaaves De usu ratiocinii mechanici in medicina (Vom Nutzen des mechanischen Vernunftschlusses in der Medizin, 1702). Ebenso vertraut ist ihm die enge Verbindung von inneren und äußeren Leiden, wie sie von Boerhaave, aber auch von Friedrich Hoffmann vertreten wurde ${ }^{9}$.

Die gemeinsame Grundlage der Medizin und Chirurgie bildet die mechanistische Deutung der Lebensvorgänge; der Schlüssel dazu ist die Anatomie. Physiologie und Chirurgie treten auch dadurch in enge Beziehung, daß sich der experimentierende Arzt chirurgischer Techniken bedient: der Tierversuch ist eine chirurgische Operation.

Die Iatromechaniker insistieren umso mehr auf der Wichtigkeit und dem Nutzen der Zergliederungskunst, als von Seiten Stahls und seiner Anhänger die Anatomie, besonders die «Anatomia recentior curiosa», einer heftigen Kritik unterzogen wird. Was nützt dem Arzt die Kenntnis des inneren Baues 
des Muskels, wenn dieser - wie es täglich vorkommt - verletzt ist? Kann er etwa die einzelnen Fasern wieder zusammensetzen? Mitnichten! Stahl fährt in seiner Theoria medica vera fort:

«Ich bestreite nämlich, daß aus dem ganzen Bau des Körpers, aus dem Bau und der Textur der organischen Teile, nicht nur bezüglich ihrer Mechanik, sondern auch ganz allgemein, etwas hervorgeht, was dem Arzt wirklich dient, was er unbedingt wissen müßte. Mehr noch: Ich leugne, daß derartige [anatomische] Kenntnisse dem Arzt als solchem strenggenommen auch nur ein bißchen nützen zur Erreichung seines Zieles, nämlich zu heilen, wiederherzustellen, ... zu verhüten und wiedergutzumachen». ${ }^{10}$

Nach Heisters Überzeugung ist die Anatomie dagegen für den Arzt und den Chirurgen, was der Kompaß für den Steuermann eines Schiffes, wie der Leser den einleitenden Paragraphen des vielbenützten Lehrbuchs entnehmen konnte ${ }^{11}$. In seiner Helmstedter Antrittsrede von 1720 gab Heister einen Überblick über den eindrücklichen Zuwachs des anatomischen Wissens in den ersten zwei Dezennien des 18. Jahrhunderts ${ }^{12}$. Heisters Schüler, der Schlesier Johann Carl Hahn, verteidigte 1728 in seiner Dissertation die subtile Anatomie gegen ihre Verächter ${ }^{13}$.

Hahn räumt ein, vorsichtige Anatomen seien nicht so arrogant zu glauben, daß mittels der Scientia anatomica alle Krankheiten heilbar würden. Der Medicus rationalis gewinne aber durch die Anatomie in sehr vielen dunkeln inneren und äußeren Krankheiten, besonders aber in chirurgischen Leiden eine klarere Kenntnis, so daß er bessere und sicherere Heilanzeigen und Gründe für sein Handeln daraus ableiten könne, was für zahlreiche Fälle (z.B. Star, Steinleiden, Hernien usw.) belegt wird. Viele anatomische Entdeckungen würden übrigens erst später praktisch verwertbar, wenn andere Forscher sie aufgriffen. Schließlich sei es verderblich, die Studenten durch Scheinargumente vom Studium der Anatomie abzuhalten.

So wie Heister im Compendium anatomicum als erstes Ziel der Anatomie die «Gloria Dei» nennt, so schließt auch Hahn mit der Feststellung: «Deum in minimis esse maximum» (Gott ist im kleinsten am größten); verwerflich ist es, den Menschen, das vollkommenste Geschöpf, nicht genauer kennen zu wollen.

Im Gebäude der Physikotheologie ist die Anatomie eine der tragenden Säulen. Die mechanistische Konzeption der Physiologie garantiert einen freien Raum für die kausal-analytische Forschung, sofern zuvor dem Erbauer der Maschine und dessen Stellvertretern auf Erden die gebührende Reverenz erwiesen wurde. 
Die mechanistische Medizin verleiht der Chirurgie eine besondere Würde. Heister läßt keine Gelegenheit unbenutzt, mit Celsus festzustellen, die Chirurgie sei der älteste und gewisseste Teil der Medizin. Entscheidend ist für ihn der mechanische Eingriff, die Reposition, die Ruhigstellung, die Vereinigung der Wundränder, die Entfernung der fehlerhaften Teile: die Chirurgie ist die «mechanische Medizin» par excellence.

Für Stahl liefert die Chirurgie den Gegenbeweis:

«Die Verletzungen der Solida lassen kaum eine ausführliche medizinische Theorie zu, die einer kunstreichen Wirksamkeit dienen könnte, denn der Baumeister der Wiederherstellung der festen Teile ist überall allein die Natur. Keine Kunst ist in der Lage, auch nur einen Punkt, geschweige denn einen Faden oder eine Faser im Körper zusammenzufügen ...» ${ }^{14}$

Johannes Juncker (1679-1759) betont in seiner nach Stahlschen Grundsätzen verfaßten Chirurgie, wie wichtig es für den Chirurgen sei, das «zeitliche Moment» zu erkennen, «das von der Natur in der spontanen Wiederherstellung der Struktur angewendet werde.» Insbesondere sei es Sache des Arztes, bei Verletzungen zu beurteilen, «was durch instrumentelle Hilfe zu korrigieren und was von der Heilwirkung der Natur allein zu erhoffen sei». ${ }^{15}$

Heister hält gegen Stahls Natur- bzw. Seelenwirkung fest:

«... GOtt hat die Theile unsers Cörpers nach seiner Weisheit also gebauet, daß die zerbrochenen Knochen durch ihren eignen Saft, woraus zuerst ein Knorpel wird, wieder zusammenwachsen. Desgleichen sind auch die Säfte in den Muskeln und weichen Theilen nach ihrer Natur eingerichtet, also daß man nicht nöthig hat, hier mit den Stahlianern die zweifelhafte Vorsorge der Seele zu Hülfe zu nehmen, und ihr eine Weisheit und Kraft, welche sie nicht hat, zuzuschreiben, indem alles gar füglich aus dem Bau dieser Theile, welche sowohl die Absonderung als Heilung verrichten, kan erkläret und begriffen werden» (§62).

Ganz im Sinne Heisters hatte 1729 der Wittenberger Chirurgieprofessor Christian Gottfried Stenzel (1698-1748) von der Ohnmacht der Stahlschen Natur in der Chirurgie gesprochen ${ }^{16}$.

Ärzte unterschiedlicher theoretischer Richtung stimmten darin überein, daß die Chirurgie in den Händen der Barbiere in traurigen Verfall geraten sei. Aus beiden Lagern ertönte der Ruf nach der Wiedervereinigung der Wundarzneikunst mit der Medizin, nach besserer Ausbildung der Ärzte in Anatomie und Chirurgie.

Juncker möchte die Chirurgen als medizinische Hilfspersonen weiterhin mit der Chirurgia manualis oder mechanica betrauen, unter Aufsicht des 
Arztes selbstverständlich, dem allein die Chirurgia medica, d.h. die internistische Behandlung chirurgischer Leiden, zukommt ${ }^{17}$.

Viel weiter geht - atypisch für einen Stahlianer, als der er später bekannt wurde -... Andreas Ottomar Goelicke (1670/71-1744) im Jahre 1709, kurz nachdem er von einem einjährigen Studienaufenthalt in Holland nach Halle zurückgekehrt war. Er schlug vor, durch systematische Ausbildung der Mediziner in Anatomie und Chirurgie den operierenden Barbieren nach und nach das Wasser abzugraben und so de facto die medizinischen Disziplinen wieder $z u$ vereinigen ${ }^{18}$.

Ähnlich weit gehende Ideen unterbreitete 1731 Heisters Doktorand Georg Christian Maternus ${ }^{19}$. Er war überzeugt, daß die für die Ausübung der «Chirurgiatrik» notwendige «Scientia physiologico-pathologico-anatomico-pharmaceutica» für den Barbier (tonsor) unerreichbar ist. Wie sollte nach seiner Meinung die Vereinigung von Medizin und Chirurgie verwirklicht werden? Nach dreijährigem Studium der theoretischen Medizin sollten vier oder mehr Jahre darauf verwendet werden, «die Theorie auf die Praxis anzuwenden und die Instrumental-Operationen auszuführen». «Würde damit die Res publica medicorum nicht ein anderes Gesicht erhalten?» Nun zu den Einwänden: Wo sollte dieser Unterricht stattfinden? Es gibt Orte, an denen in einem einzigen Jahr mehr Operationen vorkommen, als sie ein Achtzigjähriger in seiner ganzen Praxis erleben kann, nämlich die öffentlichen Spitäler, z. B. in Wien, Paris, London, Berlin, oder in Amsterdam, Nürnberg, Breslau. Die erforderlichen Kosten würden dem öffentlichen Wohl zugute kommen. Die Barbiere würden keineswegs zu Bettlern, sie fänden in ihren Scherstuben genug zu tun. Die Vorurteile der Ärzte gegen die Chirurgie wären überwindbar. Der Einwand, daß die Barbiere ihre Sache doch nicht so schlecht machten, wird widerlegt durch die Tatsache, daß sie immer wieder gefährliche, ja giftige Medikamente verschreiben, was bei ihrer Ignoranz nicht verwunderlich ist.

Im Gegensatz zu anderen Systemen bot Heisters Iatromechanik für die Aufwertung der Chirurgie und des chirurgischen Eingriffes eine theoretische Grundlage: die Anatomie und eine daraus abgeleitete, den Gesetzen der Naturwissenschaften unterworfene Physiologie. Trotzdem war-abgesehen von einzelnen Ärzten, die nach Heisters Vorbild auch chirurgisch praktizierten - den Vorschlägen von Goelicke und Maternus/Heister wohl kein Erfolg beschieden. Die Iatromechanik verlor im Laufe des 18. Jahrhunderts an Überzeugungskraft ${ }^{20}$; Heisters Ausfall gegen Stahl ist bereits ein Rückzugsgefecht. Wie nüchtern derartige Wiedervereinigungspläne in den Augen der 
zeitgenössischen Praktiker erschienen, formulierte 1727 der Hannoveraner Arzt Gerhard Stoer: Jedermann muß vermuten, daß es den Ärzten nur um den Geldbeutel geht, wenn sie die Praxis der Chirurgen (und der Apotheker, die Goelicke ebenfalls ausrotten wollte) an sich reißen. Ferner zeugt es nicht von Nächstenliebe, wenn man auf Kosten der schlecht ausgebildeten Barbiere profitieren will, anstatt ihnen Belehrung anzubieten, Belehrung, die immerhin verschiedenen Orts von «Hohen Häuptern» verordnet worden ist. Wenn die Ärzte hier ihre Mitwirkung verweigern, gerät ihre staatsbürgerliche Einstellung in ein schiefes Licht. Im übrigen sorgen die Medizinalordnungen dafür, daß jedem das Seine gesichert bleibt ${ }^{21}$.

\section{Anmerkungen}

${ }^{1}$ Fossel, Viktor, Studien zur Geschichte der Medizin, Stuttgart 1909 (S.111-152: Lorenz Heister); Gruber, Georg B., Lorenz Heister und die Früh-Entwicklung der Chirurgie in Deutschland. Niedersächsisches Arzteblatt 1958, S.282-288; ders. Neue Deutsche Biographie, Bd.8, S. 458 f.; Leporin, Christian Polykarp, Ausführlicher Bericht vom Leben und Schrifften Des ... Laurentii Heisteri, Quedlinburg 1725; Wernsdorf, Johann Christian, Memoria ... Laurentii Heisteri ..., Helmstadii (1758) (mit Bibliographie Heisters). Vergleiche: Fischer, Georg, Chirurgie vor 100 Jahren, Leipzig 1876, S. 158-170.

2 Soviel ich sehe, wurde diese Schrift bisher lediglich von Robert Herrlinger kurz gewürdigt: Heister contra Stahl, Eine zeitgenössische Polemik um Grundauffassungen der barocken Medizin. Ärztliche Praxis 3, 1951, Nr.49. - Meine Zitate stammen aus dem im Text genannten Handbuch ... bzw. Compendium ...

${ }^{3}$ Gottlieb, Bernward Josef, Bedeutung und Auswirkungen des hallischen Professors ... Georg Ernst Stahl auf den Vitalismus des XVIII. Jahrhunderts, insbesondere auf die Schule von Montpellier. Nova Acta Leopoldina, N. F. Bd. 12, Nr. 89, S.425-502. - King, Lester S., The Philosophy of Medicine. The Early Eighteenth Century, Cambridge .. 1978; Rothschuh, Karl E., Konzepte der Medizin in Vergangenheit und Gegenwart, Stuttgart (1978).

${ }^{4}$ Boerhaave, Herman, Institutiones medicae ... Leiden 1708; Heister beruft sich ferner auf folgende Autoren: Giovanni Alfonso Borelli (1608-1679), Lorenzo Bellini (1634-1704), Nicolaus Steno (1638-1686), Richard Lower (1631-1691), Archibald Pitcairne (1652-1713), Friedrich Hoffmann (1660-1742), Johann Gottfried von Berger (1659-1736), Domenico Guglielmini (1655-1710), Giovanni Maria Lancisi (1654-1720), James Keill (1673-1719), Richard Mead (1673-1754), Pietro Antonio Michelotti (1673-1740), Giovanni Battista Mazzini (1677-1743), Johann Friedrich Schreiber (1705-1760), Georg Erhard Hamberger (1697-1755).

${ }^{5}$ Heister, Lorenz, Compendium institutionum sive fundamentorum medicinae ... Editio altera ..., Helmstedt 1745, S. 8; Heisters Eintreten für die Iatromechanik und seine Polemik gegen Stahl sind hier im Vergleich zur 1. Auflage (1736) deutlich verstärkt. 
${ }^{6}$ Vgl. dazu: Neuburger, Max, Die Lehre von der Heilkraft der Natur im Wandel der Zeiten, Stuttgart 1926; Robert Boyle setzte 1682 «Natur» mit «Mechanismus» gleich (S.51 ff.); Heisters Stellung wird S. 75 f. gewürdigt.

${ }^{7}$ Vgl. dazu die unter Heisters Präsidium verteidigte Dissertation: Rochlitz, Ioh. Theophilus Hugo, De medico naturae domino, Helmstedt 1730. Natur und Arzt werden hier mit Pferd und Reiter verglichen.

${ }^{8}$ Heisters Bibliographie in Wernsdorf (Anm. 1).

9 Boerhaave bespricht die chirurgisehen Krankheiten ausführlich in den Aphorismi de cognoscendis et curandis morbis; zu Friedrich Hoffmann vgl. z. B. das Vorwort zur Gesamtausgabe, Opera omnia physico-medica, Bd.1, Genf 1761: De differente artis medicae \& Medicorum conditione ac statu \& Criteriis boni ac periti Medici, S. XXXf.

${ }^{10}$ Stahl, Georg Ernst, Theoria medica vera, ed. Ludovicus Choulant, Leipzig 1831-1833, Bd. 1, S. 69 .

1 Heister, Lorenz, Compendium anatomicum totam rem anatomicam brevissime complectens, Altorf und Nürnberg 1719, $\S 8$; dieselbe Formulierung findet sich auch in den späteren Auflagen.

12. Heister, Lorenz, Oratio de incrementis anatomiae in hoc seculo XVIII. ..., Wolfenbüttel 1720

${ }^{13}$ Hahn, Ioh. Carolus, De anatomes subtilioris utilitate Praeside Laurentio Heistero ..., Helmstedt 1728.

14 Stahl (Anm. 10), Bd.3, S.377.

${ }_{15}$ Juncker, Johannes, Conspectus chirurgiae tam medicae, methodo Stahliana conscriptae; quam instrumentalis ..., Editio secunda, Halle 1731, S. $10 \mathrm{f}$.

${ }^{16}$ Stenzel, Christian Gottfried, De naturae Stahlianae in chirurgia impotentia ..., Wittenberg 1729.

17 Juncker (Anm. 15), S. 10 f.; vgl. dazu: Schweizer, Georg Wilhelm, De medicinae et chirurgiae perpetuo nexu ... sub praesidio Dn. Georgii Ernesti Stahl .... Halle 1705. Schweizer fordert für den Chirurgen eine solide medizinische Ausbildung; der Arzt muß vor allem die chirurgische Theorie beherrschen.

${ }_{18}$ Goelicke, Andreas Ottomar, De mutilo medicinae corpore resarciendo, per chirurgiam et pharmaciam postliminio revocandas ... respondens Christianus Knaut, Halle 1709.

19 Maternus, Georg Christian, De chirurgia cum medicina necessario coniugenda ... Praeside Laurentio Heistero .... Helmstedt 1732.

20) Vgl. dazu: Brown, Theodore M., From Mechanism to Vitalism in Eightennth-Century English Physiology. Journal of the History of Biology 7, 1974, S. 179-216; Toelner, Richard, Mechanismus - Vitalismus: ein Paradigmawechsel? Testfall Haller. In: Diemer, Alwin, Die Struktur wissenschaftlicher Revolutionen und die Geschichte der Wissenschaften. Meisenheim am Glan 1977, S.61-72; Luyendijk-Elshout, A. M., Nechanicisme contra Vitalisme. De school van Herman Boerhaave en de beginselen van het leven. Tijdschrift voor de geschiedenis der geneeskunde, natuuruetenschappen, wiskunde en techniek 5, 1982, Nr.1, S. 16-26.

21 Stoer, Gerhard, Untersuchung der Frage: Ob es nöthig, nützlich, billig ... sey, die Praxin der Medicin, Chirurgie- und Apotheker-Kunst in einer Persohn zu vereinigen .... Helmstedt 1727 (mit einer deutschen Übersetzung von Goclicke [Anm. 18]). 


\section{Summary}

In his Dissertatio de medicinae mechanicae praestantia Laurentius Heister (1683-1758), the famous German surgeon and physician, revealed himself as a firm supporter of the iatromechanical concept of life in argumenting against G.E.Stahl's views. It is proposed that this iatromechanical concept helped to raise the general esteem of surgery and fostered the idea to reunite surgery with medicine.

PD Dr. med. Urs Boschung

Medizinhistorisches Institut der Universität

Rämistraße 71

CH-8006 Zürich 